\title{
Adaptive Sliding Mode Controller Design for Projective Synchronization of Different Chaotic Systems with Uncertain Terms and External Bounded Disturbances
}

\author{
Shijian Cang, ${ }^{1}$ Zenghui Wang, ${ }^{2}$ and Zengqiang Chen ${ }^{3}$ \\ ${ }^{1}$ Department of Industry Design, Tianjin University of Science and Technology, Tianjin 300457, China \\ ${ }^{2}$ Department of Electrical and Mining Engineering, University of South Africa, Florida 1710, South Africa \\ ${ }^{3}$ Department of Automation, Nankai University, Tianjin 300071, China \\ Correspondence should be addressed to Shijian Cang; sj.cang@gmail.com and Zenghui Wang; wangzengh@gmail.com
}

Received 22 January 2013; Accepted 1 June 2013

Academic Editor: Constantinos Siettos

Copyright (C) 2013 Shijian Cang et al. This is an open access article distributed under the Creative Commons Attribution License, which permits unrestricted use, distribution, and reproduction in any medium, provided the original work is properly cited.

\begin{abstract}
Synchronization is very useful in many science and engineering areas. In practical application, it is general that there are unknown parameters, uncertain terms, and bounded external disturbances in the response system. In this paper, an adaptive sliding mode controller is proposed to realize the projective synchronization of two different dynamical systems with fully unknown parameters, uncertain terms, and bounded external disturbances. Based on the Lyapunov stability theory, it is proven that the proposed control scheme can make two different systems (driving system and response system) be globally asymptotically synchronized. The adaptive global projective synchronization of the Lorenz system and the Lü system is taken as an illustrative example to show the effectiveness of this proposed control method.
\end{abstract}

\section{Introduction}

The cooperative behavior of coupled nonlinear oscillators is of interest in connection with a wide variety of different phenomena in physics, engineering, biology, and economics. For example, systems of coupled nonlinear oscillators may be used to explain how different sectors of the economy adjust their individual commodity cycles relative to one another through the exchange of goods and capital units or via aggregate signals in the form of varying interest rates or raw materials prices. As part of the cooperative behaviors, the synchronization plays very important role in many applications. Synchronization occurs when oscillatory (or repetitive) systems via some kind of interaction adjust their behaviors relative to one another so as to attain a state where they work in unison [1], since the individual oscillators display chaotic dynamics in many cases and it is very important to analyze the synchronization of chaotic systems.

Synchronization of two coupled chaotic systems has attracted much attention for both theoretical studies and practical applications since the synchronization of the different chaotic systems was observed by Pecora et al. in 1997 [2]. Many investigations have been devoted to synchronization due to its potential application in many fields recently, such as security communication $[3,4]$, information processing [5, 6], and biological systems [7]. Many different synchronization strategies of different chaotic systems have been developed. Beside the generalized synchronization $[8$, 9], there are some other types of synchronization which are also very interesting and useful, like phase synchronization $[10,11]$, antiphase synchronization $[12,13]$, complete synchronization [14, 15], and lag synchronization [16, 17]. In 1999, a new synchronization observed by Mainieri and Rehacek in partially linear chaotic systems is called projective synchronization [18]. The dynamical behavior of projective synchronization refers to that two identical or different systems which are synchronized up to a constant scaling factor.

So far, great progress has been made in the research of projective synchronization among all types of synchronization because of its adjustable proportionality between the synchronized dynamical states $[19,20]$. Xu et al. designed a control scheme to manipulate the scaling factor onto any 
desired value [21]. Wen realized full-state projective synchronization by using an observer-based control [22]. Sudheer and Sabir designed a coupling function for unidirectional coupling in identical and mismatched oscillators to realize function projective synchronization through openplus-closed-loop coupling method [23]. In addition, Peng and Jiang realized generalized projective synchronization of a class of fractional-order chaotic systems via a scalar transmitted signal [24]. When projective synchronization is applied to secure communications, the binary sequences can be extended to $\mathrm{N}$-nary sequences in digital communication for achieving higher speed [25], which would exert a great influence on communication.

To achieve projective synchronization of identical or nonidentical chaotic systems with different initial conditions, many effective control methods have been proposed, such as linear and nonlinear feedback control [26], adaptive control [27], impulsive control [28], and sliding mode control [29]. With these control schemes, many dynamical systems, such as the Lorenz system, the Chen system, the Rössler system, and some other chaotic or hyperchaotic systems [30, 31], with known or unknown parameters, are all synchronized by various control methods based on Lyapunov stability theory. Sliding mode control is a variable structure control algorithm as it alters the dynamics of a nonlinear system by the application of a high-frequency switching control. In view of the simple structure, good robustness, and high reliability, sliding mode control is widely applied to motion control in engineering, especially used in determinacy system with an accurate mathematical model [32]. When the dynamical system has been precisely modeled, this control method is an effective way to realize the projective synchronization of various dynamical systems. Mou et al. and Yang et al. provided an adaptive sliding mode control method to achieve the generalized synchronization of integral-order and fractionalorder chaotic dynamics with fully unknown parameters [33, 34]. However, the existing synchronizations were realized for only identical structure chaotic systems, and these methods cannot be directly applied to the systems with uncertain terms and disturbances which are the most cases in the applications.

In view of its great potential value in secure communication, the projective synchronization is increasingly becoming a very important research topic in the fields of synchronization and control of chaos. Since the system structure of the projective synchronization belongs to driving-response type (master-slave), the driving system and the response system synchronize up proportionally to a constant scaling factor by using a proper control scheme. Therefore, it is desirable to achieve the projective synchronization of nonidentical chaotic systems with unknown parameters, uncertain terms, and bounded external disturbances via adaptive sliding mode controller. This paper mainly focuses on the projective synchronization of nonidentical structure chaotic systems with fully unknown parameters, uncertain terms, and external bounded disturbances. Based on the Lyapunov stability theory, a kind of adaptive sliding mode controller is designed to achieve projective synchronization of two nonidentical structure chaotic systems. Here, the parameters of the response system are fully unknown, and there are uncertain terms and bounded external disturbances in the response system. Moreover, the complete projective synchronization and the antiphase projective synchronization can be realized by adjusting the scaling factor.

The remainder of this paper is organized as follows. Section 2 presents the general theory of projective synchronization. In Section 3, an adaptive sliding mode controller is proposed and the robust stability is analyzed. Synchronization analysis of the Lorenz system and the Lü system is given in Section 4, and finally, Sections 5 and 6 provide the numerical simulations and concluding remarks of this study, respectively.

\section{General Theory of Projective Synchronization}

The main problem discussed in this paper is projective synchronization, which will be implemented by driving-response scheme. The driving system can be described by the nonlinear differential equation

$$
\dot{x}=f(x, \phi, t)
$$

where the observable state variable $x \in R^{n}$ is an $n$-dimensional column vector, $\phi \in R^{l}$ represents an $l$-dimensional parameter vector, and $f(\cdot) \in R^{n}$ is a continuous or noncontinues nonlinear function vector.

The response system can be expressed as

$$
\dot{y}=g(y, \theta, t)+u(t)
$$

where $y \in R^{n}$ denotes the observable state variables of the response system (2), $\theta \in R^{m+1}$ represents an $(m+1)$ dimensional known or unknown parameter vector, $m$ is the number of unknown parameters, $g(\cdot) \in R^{n}$ is a function vector similar with $f(\cdot)$, and $u(t) \in R^{n}$ is an $n$-dimensional control input vector which can be used to realize projective synchronization of the systems (1) and (2).

When the tracking error is defined as $e_{i}=y_{i}-\kappa x_{i}(i=$ $1,2, \ldots, n)$, the synchronization error differential equation can be obtained as

$$
\dot{e}=\dot{y}-\kappa \dot{x}=g(y, \theta, t)-\kappa f(x, \phi, t)+u(t) .
$$

Here, the parameter $\kappa$ is a scaling factor, which can adjust the synchronization proportion of the systems (1) and (2).

In order to achieve the synchronization of the driving system (1) and the response system (2), the control input $u(t)$ needs to satisfy

$$
\lim _{t \rightarrow \infty}\|e(t)\| \longrightarrow 0
$$

Using a proper control scheme, this kind of synchronization is called projective synchronization if $\lim _{t \rightarrow \infty}\|e(t)\| \rightarrow 0$ by varying the scaling factor $\kappa$.

Projective synchronization includes several kinds of synchronization. Complete synchronization and antiphase synchronization are two special cases corresponding with scaling factor $\kappa=1$ and -1 , respectively. 
In view of the structure of the systems (1) and (2), the projective synchronization can be divided into identical structure projective synchronization and nonidentical structure projective synchronization. In this paper, we focus on the projective synchronization of two nonidentical structure systems, which means that the driving system (1) and the response system (2) are not same, and the identical structure projective synchronization can be looked as a specific case of nonidentical structure projective synchronization.

\section{Adaptive SMC Design and Robust Stability Analysis}

In the most of the practical applications, the parameters of the response system are not known, and there are uncertain terms and external bounded disturbances in the response system. Therefore, it is necessary to consider this practical matter. In the following sections, this kind of response systems will be applied to the design and analysis of synchronization. This kind of response system can be described as

$$
\dot{y}=g(y, \theta, t)+\Delta g(y, \theta, t)+d(t)+u(t),
$$

where parameter vector $\theta \in R^{m+1}$ is unknown, $m$ is the number of unknown parameters, $\Delta g(y, \theta, t)$ denotes uncertain and bounded function vector, and $d(t)$ is the unknown and bounded external disturbances, such as DC signal, AC signal, any kind of bounded noise signals, and chaotic signal and $u(t)$ represents the control input vector. Hence, $\Delta g(y, \theta, t)$ and $d(t)$ should meet the following conditions:

$$
\begin{gathered}
\|d(t)\| \leq D(t) \in L_{\infty}, \\
\|\Delta g(y, \theta, t)\| \leq H(y, t) \in L_{\infty},
\end{gathered}
$$

where $D(t)$ and $H(y, t)$ are two known upon bound of external disturbance and uncertain function, respectively, which are always set to be constants without loss of generality. In general, $g(y, \theta, t)$ can be described by the linear parameterization structure

$$
g(y, \theta, t)=G(y, t) \theta,
$$

where the elements of matrix $G(y, t) \in R^{n \times(m+1)}$ and parameter vector $\theta_{i}(i=1,2, \ldots, n)$ can be one-order, high-order, or constant terms. Therefore, the response system (5) can be rewritten as

$$
\dot{y}=G(y, t) \theta+\Delta g(y, \theta, t)+d(t)+u(t) .
$$

Using the definition of tracking error, one can further obtain the synchronization error differential vector

$$
\begin{aligned}
\dot{e}= & \dot{y}-\kappa \dot{x}=G(y, t) \theta+\Delta g(y, \theta, t) \\
& +d(t)-\kappa f(x, \phi, t)+u(t),
\end{aligned}
$$

where $e=\left[e_{1}, e_{2}, \ldots, e_{n}\right]^{T}$.

Definition 1. For any initial conditions of the systems (1) and (8), the zero solution of the error system (9) is globally stable if the motion trajectory of the error dynamical system (9) satisfies $\left|e_{i}\right| \rightarrow 0(i=1,2, \ldots, n)$ as $t \rightarrow+\infty$. The drivingresponse systems (1) and (8) are globally projective synchronization when the scaling factor $\kappa \neq 0$. Specially, for $\kappa=1$, it is called global complete synchronization, and it is called globally antiphase synchronization for $\kappa=-1$.

We construct an $n$-dimensional sliding mode surface $s=$ $\left[s_{1}, s_{2}, \ldots, s_{n}\right]^{T}$ with $s_{i}=\mu_{i} e_{i}(i=1,2, \ldots, n)$ and adopt a constant-velocity reaching law as a control scheme to realize globally projective synchronization of two nonidentical structure systems. Here, an $n$-dimensional matrix of gain adjustment $\Gamma$ can be used to denote the parameters $\mu_{i}(i=$ $1,2, \ldots, n)$, that is, $\Gamma=\operatorname{diag}\left(\mu_{1}, \mu_{2}, \ldots, \mu_{n}\right)$. Further, the $n$ dimensional sliding mode surface $s$ can be represented as $s=\Gamma e$.

Theorem 2. For the system (9), if the control input is designed as

$$
u(t)=-G(y, t) \bar{\theta}+\kappa f(x, \phi, t)-\rho(t) \operatorname{sgn}(s),
$$

the zero solution of the error system (9) is globally stable when $\rho(t)=H(y, t)+D(t)+\eta(\eta>0)$. Here, $\bar{\theta}$ is the estimated vector of the unknown parameter $\theta$ of the system (8), $\rho(t)$ denotes the constant-velocity of the trajectory inclining to the switch surface $s=0$, and the expression $\operatorname{sgn}(s)$ is a sign function vector. In other words, the systems (1) and (8) can asymptotically achieve projective synchronization by using the control law (10) when $\rho(t)=H(y, t)+D(t)+\eta(\eta>0)$.

Proof. Construct the positive Lyapunov function

$$
V(s, \theta)=\frac{1}{2} s^{T} s+\frac{1}{2} \widetilde{\theta}^{T} \widetilde{\theta},
$$

where $\widetilde{\theta}=\theta-\bar{\theta}$ is the estimation error vector of the parameter vector. Then, one can obtain $\dot{\tilde{\theta}}=-\dot{\bar{\theta}}$ as $\theta$ is a constant parameter vector for a certain response system. Taking time derivative of (11) along the solution of error dynamics (9) and control input (10),

$$
\begin{aligned}
\dot{V}(\cdot)= & s^{T} \dot{\boldsymbol{s}}+\widetilde{\theta}^{T} \dot{\tilde{\theta}} \\
= & s^{T} \Gamma \dot{e}+\widetilde{\theta}^{T} \dot{\tilde{\theta}} \\
= & s^{T} \Gamma[G(y, t) \theta+\Delta g(y, \theta, t)+d(t) \\
& -\kappa f(x, \phi, t)+u(t)]+\widetilde{\theta}^{T} \dot{\tilde{\theta}} \\
= & s^{T} \Gamma[G(y, t) \widetilde{\theta}+\Delta g(y, \theta, t)+d(t) \\
& -\rho(t) \operatorname{sgn}(s)]+\widetilde{\theta}^{T} \dot{\tilde{\theta}}
\end{aligned}
$$




$$
\begin{aligned}
& =s^{T} \Gamma[\Delta g(y, \theta, t)+d(t)-\rho(t) \operatorname{sgn}(s)] \\
& +\widetilde{\theta}^{T} G(y, t)^{T} \Gamma^{T} \mathcal{s}-\widetilde{\theta}^{T} \dot{\bar{\theta}} \\
& =\left\{\begin{array}{rr}
s^{T} \Gamma[\Delta g(y, \theta, t)+d(t)-(H(y, t)+D(t)+\eta) v] \\
+\widetilde{\theta}^{T}\left(G(y, t)^{T} \Gamma^{T} s-\dot{\bar{\theta}}\right) & s_{i} \geq 0, \quad i=1,2, \ldots, n \\
s^{T} \Gamma[\Delta g(y, \theta, t)+d(t)+(H(y, t)+D(t)+\eta) \nu] \\
+\widetilde{\theta}^{T}\left(G(y, t)^{T} \Gamma^{T} s-\dot{\bar{\theta}}\right) & s_{i}<0, \quad i=1,2, \ldots, n
\end{array}\right. \\
& \leq-\eta\left|s^{T} \Gamma \nu\right|+\widetilde{\theta}^{T}\left(G(y, t)^{T} \Gamma^{T} s-\dot{\bar{\theta}}\right),
\end{aligned}
$$

where $v$ is an $n$-dimensional vector with all entries equal to one, namely, $v=[1,1, \ldots, 1]^{T}$. If the parameter identification update law is as follows:

$$
\dot{\bar{\theta}}=G(y, t)^{T} \Gamma^{T} s=G(y, t)^{T} \Gamma^{T} \Gamma e .
$$

One can further obtain

$$
\dot{V}(\cdot) \leq-\eta\left|s^{T} \Gamma \nu\right|=-\eta\left(\sum_{i=1}^{n}\left|\mu_{i} s_{i}\right|\right) \leq 0 .
$$

Then,

$$
\begin{gathered}
V(\cdot) \geq 0, \\
\dot{V}(\cdot) \leq 0 \longrightarrow s_{i} \in L_{\infty} \\
\longrightarrow e_{i} \in L_{\infty} \stackrel{x_{i} \in L_{\infty}}{\longrightarrow} y_{i} \in L_{\infty} \stackrel{\text { expression }(14)}{\longrightarrow} \dot{\bar{\theta}}_{i} \in L_{\infty}, \\
V(\cdot) \geq 0 \\
\dot{V}(\cdot) \leq 0 \longrightarrow \widetilde{\theta} \in L_{\infty} \\
\longrightarrow(\bar{\theta} \longrightarrow \theta) \stackrel{\theta \in L_{\infty}}{\longrightarrow} \bar{\theta} \in L_{\infty}, \\
x_{i}, y_{i}, s_{i}, \bar{\theta}_{i}, \rho \stackrel{\text { expression }(10)}{\longrightarrow} u_{i} \in L_{\infty} \\
\dot{x}_{i}, \dot{e}_{i} \stackrel{\dot{e}_{i}=\dot{y}_{i}-\kappa \dot{x}_{i}}{\longrightarrow} \dot{y}_{i} \in L_{\infty} .
\end{gathered}
$$

In view of this, we define a new function $f(\cdot)=\sum_{i=1}^{n} k_{i} e_{i}^{2}$, and the following equation (19) can be determined by derivative operation:

$$
\dot{f}(\cdot)=2 \sum_{i=1}^{n} k_{i} e_{i} \dot{e}_{i} \in L_{\infty} .
$$

As can be seen from (15) to (18), the estimated parameters, error variables, and state variables are bounded. Based on (15), (16), (19), and the Barbalat lemma, $\left|e_{i}\right| \rightarrow 0(i=$ $1,2, \ldots, n)$ as $t \rightarrow+\infty$, and the error dynamical system (9) is asymptotically stable at the origin. Therefore, this projective synchronization theorem was proved.

\section{Projective Synchronization of the Lorenz System and the Lü System}

In this section, the projective synchronization of the Lorenz system and the Lü system is used as an example to validate the proposed adaptive control technique in Section 3, where the Lorenz system is regarded as the driving system, and another with control inputs $u(t)$, unknown system parameters $\theta$, uncertain terms $\Delta g(y, \theta, t)$, and bounded external disturbances $d(t)$ acts as the response system.

The Lorenz system [35] is given by

$$
\begin{gathered}
\dot{x}_{1}=a_{1}\left(x_{2}-x_{1}\right), \\
\dot{x}_{2}=b_{1} x_{1}-x_{2}-x_{1} x_{3}, \\
\dot{x}_{3}=x_{1} x_{2}-c_{1} x_{3},
\end{gathered}
$$

which has a typical butterfly chaotic attractor and captures lots of features of chaotic systems when the parameters $a_{1}=$ $10, b_{1}=28$, and $c_{1}=8 / 3$. The Lü system [36] is a typical chaos anticontrol model, which is a chaotic system between the Lorenz system and the Chen system in the unified Lorenz system family. The nonlinear differential equations of the Lü system are

$$
\begin{aligned}
& \dot{y}_{1}=a_{2}\left(y_{2}-y_{1}\right), \\
& \dot{y}_{2}=b_{2} y_{2}-y_{1} y_{3}, \\
& \dot{y}_{3}=y_{1} y_{2}-c_{2} y_{3} .
\end{aligned}
$$

There exists a chaotic attractor in the Lü system when the parameters $a_{2}=30, b_{2}=22$, and $c_{2}=3$. In view of (8), the controlled Lü system (21) can be rearranged as

$$
\begin{aligned}
y= & {\left[\begin{array}{l}
\dot{y}_{1} \\
\dot{y}_{2} \\
\dot{y}_{3}
\end{array}\right] } \\
= & G(y, t)\left[\begin{array}{l}
\theta_{1} \\
\theta_{2} \\
\theta_{3} \\
\theta_{4}
\end{array}\right]+\left[\begin{array}{l}
\Delta g_{1}(y, \theta, t) \\
\Delta g_{2}(y, \theta, t) \\
\Delta g_{3}(y, \theta, t)
\end{array}\right] \\
& +\left[\begin{array}{l}
d_{1}(t) \\
d_{2}(t) \\
d_{3}(t)
\end{array}\right]+\left[\begin{array}{l}
u_{1}(t) \\
u_{2}(t) \\
u_{3}(t)
\end{array}\right],
\end{aligned}
$$

where

$$
\begin{gathered}
G(y, t)=\left[\begin{array}{cccc}
y_{2}-y_{1} & 0 & 0 & 0 \\
0 & y_{2} & 0 & -y_{1} y_{3} \\
0 & 0 & -y_{3} & y_{1} y_{2}
\end{array}\right], \\
\theta=\left[\begin{array}{l}
\theta_{1} \\
\theta_{2} \\
\theta_{3} \\
\theta_{4}
\end{array}\right]=\left[\begin{array}{c}
a_{2} \\
b_{2} \\
c_{2} \\
1
\end{array}\right] .
\end{gathered}
$$


Then, one can obtain the following error differential equations when the system (20) and (22) are the driven system and the response system, respectively:

$$
\begin{aligned}
\dot{e}= & {\left[\begin{array}{l}
\dot{e}_{1} \\
\dot{e}_{2} \\
\dot{e}_{3}
\end{array}\right] } \\
= & G(y, t)\left[\begin{array}{l}
\theta_{1} \\
\theta_{2} \\
\theta_{3} \\
\theta_{4}
\end{array}\right]+\left[\begin{array}{l}
\Delta g_{1}(y, \theta, t) \\
\Delta g_{2}(y, \theta, t) \\
\Delta g_{3}(y, \theta, t)
\end{array}\right] \\
& +\left[\begin{array}{l}
d_{1}(t) \\
d_{2}(t) \\
d_{3}(t)
\end{array}\right]+\left[\begin{array}{l}
u_{1}(t) \\
u_{2}(t) \\
u_{3}(t)
\end{array}\right]-\kappa\left[\begin{array}{c}
a_{1}\left(x_{2}-x_{1}\right) \\
b_{1} x_{1}-x_{2}-x_{1} x_{3} \\
x_{1} x_{2}-c_{1} x_{3}
\end{array}\right] .
\end{aligned}
$$

One can construct a three-dimensional sliding mode surface

$$
s=\left[\begin{array}{l}
s_{1} \\
s_{2} \\
s_{3}
\end{array}\right]=\left[\begin{array}{l}
\mu_{1} e_{1} \\
\mu_{2} e_{2} \\
\mu_{3} e_{3}
\end{array}\right]=\Gamma\left[\begin{array}{l}
e_{1} \\
e_{2} \\
e_{3}
\end{array}\right],
$$

where the gain adjustment matrix $\Gamma$ is

$$
\Gamma=\left[\begin{array}{ccc}
\mu_{1} & 0 & 0 \\
0 & \mu_{2} & 0 \\
0 & 0 & \mu_{3}
\end{array}\right],
$$

and adopt a constant-velocity reaching law as a control method to achieve projective synchronization. For the error system (24), when the control input can be designed as

$$
\begin{aligned}
u(t)= & {\left[\begin{array}{l}
u_{1}(t) \\
u_{2}(t) \\
u_{3}(t)
\end{array}\right] } \\
= & -G(y, t)\left[\begin{array}{l}
\bar{\theta}_{1} \\
\bar{\theta}_{2} \\
\bar{\theta}_{3} \\
\bar{\theta}_{4}
\end{array}\right]+\kappa\left[\begin{array}{c}
a_{1}\left(x_{2}-x_{1}\right) \\
b_{1} x_{1}-x_{2}-x_{1} x_{3} \\
x_{1} x_{2}-c_{1} x_{3}
\end{array}\right] \\
& -\rho(t)\left[\begin{array}{c}
\operatorname{sgn}\left(s_{1}\right) \\
\operatorname{sgn}\left(s_{2}\right) \\
\operatorname{sgn}\left(s_{3}\right)
\end{array}\right],
\end{aligned}
$$

where $\bar{\theta}=\left[\bar{\theta}_{1}, \bar{\theta}_{2}, \bar{\theta}_{3}, \bar{\theta}_{4}\right]^{T}$ is the online estimation for unknown parameter vector $\theta=\left[\theta_{1}, \theta_{2}, \theta_{3}, \theta_{4}\right]^{T}$ of the system (22), that is,

$$
\bar{\theta}=\left[\begin{array}{c}
\bar{\theta}_{1} \\
\bar{\theta}_{2} \\
\bar{\theta}_{3} \\
\bar{\theta}_{4}
\end{array}\right]=\left[\begin{array}{c}
\bar{a}_{2} \\
\bar{b}_{2} \\
\bar{c}_{2} \\
1
\end{array}\right],
$$

hence, one can obtain

$$
\begin{aligned}
\dot{e}= & {\left[\begin{array}{l}
\dot{e}_{1} \\
\dot{e}_{2} \\
\dot{e}_{3}
\end{array}\right] } \\
= & G(y, t) \tilde{\theta}+\left[\begin{array}{l}
\Delta g_{1}(y, \theta, t) \\
\Delta g_{2}(y, \theta, t) \\
\Delta g_{3}(y, \theta, t)
\end{array}\right] \\
& +\left[\begin{array}{l}
d_{1}(t) \\
d_{2}(t) \\
d_{3}(t)
\end{array}\right]-\rho(t)\left[\begin{array}{l}
\operatorname{sgn}\left(s_{1}\right) \\
\operatorname{sgn}\left(s_{2}\right) \\
\operatorname{sgn}\left(s_{3}\right)
\end{array}\right],
\end{aligned}
$$

where

$$
\widetilde{\theta}=\left[\begin{array}{l}
\theta_{1} \\
\theta_{2} \\
\theta_{3} \\
\theta_{4}
\end{array}\right]-\left[\begin{array}{l}
\bar{\theta}_{1} \\
\bar{\theta}_{2} \\
\bar{\theta}_{3} \\
\bar{\theta}_{4}
\end{array}\right]=\left[\begin{array}{c}
a_{2}-\bar{a}_{2} \\
b_{2}-\bar{b}_{2} \\
c_{2}-\bar{c}_{2} \\
0
\end{array}\right]=\left[\begin{array}{c}
\widetilde{a}_{2} \\
\widetilde{b}_{2} \\
\widetilde{c}_{2} \\
0
\end{array}\right] .
$$

Consequently,

$$
\left[\begin{array}{c}
\dot{\tilde{\theta}}_{1} \\
\dot{\tilde{\theta}}_{2} \\
\dot{\tilde{\theta}}_{3} \\
\dot{\tilde{\theta}}_{4}
\end{array}\right]=-\left[\begin{array}{c}
\dot{\bar{\theta}}_{1} \\
\dot{\bar{\theta}}_{2} \\
\dot{\bar{\theta}}_{3} \\
\dot{\bar{\theta}}_{4}
\end{array}\right] \longrightarrow\left[\begin{array}{c}
\dot{\tilde{a}}_{2} \\
\dot{\widetilde{b}}_{2} \\
\dot{\tilde{c}}_{2}
\end{array}\right]=-\left[\begin{array}{c}
\dot{\bar{a}}_{2} \\
\dot{\bar{b}}_{2} \\
\dot{\bar{c}}_{2}
\end{array}\right] .
$$

Construct the following positive Lyapunov function:

$$
V(s, \tilde{\theta})=\frac{1}{2} s^{T} s+\frac{1}{2} \widetilde{\theta}^{T} \widetilde{\theta} .
$$

Taking time derivative of (33) along the solution of error dynamics (25) and the control input (28),

$$
\begin{gathered}
\dot{V}(\cdot)=s^{T} \dot{s}+\widetilde{\theta}^{T} \dot{\tilde{\theta}}=s^{T} \Gamma \dot{e}+\widetilde{\theta}^{T} \dot{\tilde{\theta}} \\
=s^{T} \Gamma G(y, t)\left[\begin{array}{c}
\widetilde{a}_{2} \\
\widetilde{b}_{2} \\
\widetilde{c}_{2} \\
0
\end{array}\right] \\
+s^{T} \Gamma\left[\left[\begin{array}{c}
\Delta g_{1}(y, \theta, t) \\
\Delta g_{2}(y, \theta, t) \\
\Delta g_{3}(y, \theta, t)
\end{array}\right]+\left[\begin{array}{l}
d_{1}(t) \\
d_{2}(t) \\
d_{3}(t)
\end{array}\right]\right. \\
\left.-\rho(t)\left[\begin{array}{l}
\operatorname{sgn}\left(s_{1}\right) \\
\operatorname{sgn}\left(s_{2}\right) \\
\operatorname{sgn}\left(s_{3}\right)
\end{array}\right]\right] \\
-\left[\begin{array}{llll}
\widetilde{a}_{2} & \widetilde{b}_{2} & \widetilde{c}_{2} & 0
\end{array}\right]\left[\begin{array}{l}
\dot{\bar{a}}_{2} \\
\dot{\bar{b}}_{2} \\
\dot{\bar{c}}_{2} \\
0
\end{array}\right]
\end{gathered}
$$




$$
\begin{aligned}
& =\left[\begin{array}{llll}
\tilde{a}_{2} & \tilde{b}_{2} & \tilde{c}_{2} & 0
\end{array}\right]\left[G^{T}(y, t) \Gamma^{T} s-\left[\begin{array}{c}
\dot{\bar{a}}_{2} \\
\dot{\bar{b}}_{2} \\
\dot{\bar{c}}_{2} \\
0
\end{array}\right]\right] \\
& +s^{T} \Gamma\left[\left[\begin{array}{l}
\Delta g_{1}(y, \theta, t) \\
\Delta g_{2}(y, \theta, t) \\
\Delta g_{3}(y, \theta, t)
\end{array}\right]+\left[\begin{array}{l}
d_{1}(t) \\
d_{2}(t) \\
d_{3}(t)
\end{array}\right]\right. \\
& \left.-\rho(t)\left[\begin{array}{l}
\operatorname{sgn}\left(s_{1}\right) \\
\operatorname{sgn}\left(s_{2}\right) \\
\operatorname{sgn}\left(s_{3}\right)
\end{array}\right]\right] \\
& =\left[\begin{array}{llll}
\tilde{a}_{2} & \tilde{b}_{2} & \tilde{c}_{2} & 0
\end{array}\right] \\
& \times\left[\begin{array}{ccc}
y_{2}-y_{1} & 0 & 0 \\
0 & y_{2} & 0 \\
0 & 0 & -y_{3} \\
0 & -y_{1} y_{3} & y_{1} y_{2}
\end{array}\right] \\
& \left.\times\left[\begin{array}{ccc}
\mu_{1} & 0 & 0 \\
0 & \mu_{2} & 0 \\
0 & 0 & \mu_{3}
\end{array}\right]\left[\begin{array}{c}
s_{1} \\
s_{2} \\
s_{3}
\end{array}\right]-\left[\begin{array}{c}
\dot{\bar{a}}_{2} \\
\dot{\bar{b}}_{2} \\
\dot{\bar{c}}_{2} \\
0
\end{array}\right]\right] \\
& +s^{T} \Gamma\left[\left[\begin{array}{l}
\Delta g_{1}(y, \theta, t) \\
\Delta g_{2}(y, \theta, t) \\
\Delta g_{3}(y, \theta, t)
\end{array}\right]+\left[\begin{array}{l}
d_{1}(t) \\
d_{2}(t) \\
d_{3}(t)
\end{array}\right]\right.
\end{aligned}
$$

$$
\begin{aligned}
& \left.-\rho(t)\left[\begin{array}{l}
\operatorname{sgn}\left(s_{1}\right) \\
\operatorname{sgn}\left(s_{2}\right) \\
\operatorname{sgn}\left(s_{3}\right)
\end{array}\right]\right] \\
& =\left[\begin{array}{llll}
\tilde{a}_{2} & \tilde{b}_{2} & \tilde{c}_{2} & 0
\end{array}\right] \\
& \times\left[\begin{array}{c}
\mu_{1} s_{1}\left(y_{2}-y_{1}\right)-\dot{\bar{a}}_{2} \\
\mu_{2} s_{2} y_{2}-\dot{\bar{b}}_{2} \\
-\mu_{3} s_{3} y_{3}-\dot{\bar{c}}_{2} \\
-\mu_{2} s_{2} y_{1} y_{3}+\mu_{3} s_{3} y_{1} y_{2}
\end{array}\right] \\
& +s^{T} \Gamma\left[\left[\begin{array}{l}
\Delta g_{1}(y, \theta, t) \\
\Delta g_{2}(y, \theta, t) \\
\Delta g_{3}(y, \theta, t)
\end{array}\right]+\left[\begin{array}{l}
d_{1}(t) \\
d_{2}(t) \\
d_{3}(t)
\end{array}\right]\right. \\
& \left.-\rho(t)\left[\begin{array}{l}
\operatorname{sgn}\left(s_{1}\right) \\
\operatorname{sgn}\left(s_{2}\right) \\
\operatorname{sgn}\left(s_{3}\right)
\end{array}\right]\right] .
\end{aligned}
$$

Here, the parameter update law is

$$
\begin{gathered}
\dot{\bar{a}}_{2}=\mu_{1} s_{1}\left(y_{2}-y_{1}\right)=\mu_{1}^{2} e_{1}\left(y_{2}-y_{1}\right), \\
\dot{\bar{b}}_{2}=\mu_{2} s_{2} y_{2}=\mu_{2}^{2} e_{2} y_{2}, \\
\dot{\bar{c}}_{2}=-\mu_{3} s_{3} y_{3}=-\mu_{3}^{2} e_{3} y_{3} .
\end{gathered}
$$

Using $\rho(t)=H(y, t)+D(t)+\eta(\eta>0)$ and (35), it can be concluded that

$$
\begin{aligned}
& \dot{V}(\cdot)=s^{T} \Gamma\left[\left[\begin{array}{l}
\Delta g_{1}(y, \theta, t) \\
\Delta g_{2}(y, \theta, t) \\
\Delta g_{3}(y, \theta, t)
\end{array}\right]+\left[\begin{array}{l}
d_{1}(t) \\
d_{2}(t) \\
d_{3}(t)
\end{array}\right]-(H(y, t)+D(t)+\eta)\left[\begin{array}{l}
\operatorname{sgn}\left(s_{1}\right) \\
\operatorname{sgn}\left(s_{2}\right) \\
\operatorname{sgn}\left(s_{3}\right)
\end{array}\right]\right] \\
& =\left\{\begin{aligned}
s^{T} \Gamma\left[\left[\begin{array}{l}
\Delta g_{1}(y, \theta, t) \\
\Delta g_{2}(y, \theta, t) \\
\Delta g_{3}(y, \theta, t)
\end{array}\right]+\left[\begin{array}{l}
d_{1}(t) \\
d_{2}(t) \\
d_{3}(t)
\end{array}\right]-\left[\begin{array}{l}
H(y, t)+D(t)+\eta \\
H(y, t)+D(t)+\eta \\
H(y, t)+D(t)+\eta
\end{array}\right]\right] \quad s_{i} \geq 0, \quad i=1,2,3 \\
s^{T} \Gamma\left[\left[\begin{array}{l}
\Delta g_{1}(y, \theta, t) \\
\Delta g_{2}(y, \theta, t) \\
\Delta g_{3}(y, \theta, t)
\end{array}\right]+\left[\begin{array}{l}
d_{1}(t) \\
d_{2}(t) \\
d_{3}(t)
\end{array}\right]+\left[\begin{array}{l}
H(y, t)+D(t)+\eta \\
H(y, t)+D(t)+\eta \\
H(y, t)+D(t)+\eta
\end{array}\right]\right] \quad s_{i}<0, \quad i=1,2,3
\end{aligned}\right. \\
& \leq-\eta\left(\left|\mu_{1} s_{1}(t)\right|+\left|\mu_{2} s_{2}(t)\right|+\left|\mu_{3} s_{3}(t)\right|\right) \leq 0 \text {. }
\end{aligned}
$$

The last part of this section is the proof of the projective synchronization of the Lorenz system and the Lü system using the control law (28) and the parameter update law (35). Hence, one can conclude that $e_{1}, e_{2}$, and $e_{3}$ tend to zero as $t \rightarrow+\infty, \bar{a}_{2} \rightarrow a_{2}, \bar{b}_{2} \rightarrow b_{2}$, and $\bar{c}_{2} \rightarrow c_{2}$. Therefore, the projective synchronization of the Lorenz system and the Lü system with unknown parameters, uncertain terms, and bounded external disturbances is realized by the adaptive sliding mode controller (28). In view of (15) to (19), one can obtain

$$
\begin{aligned}
& e_{1}, e_{2}, e_{3}, y_{1}, y_{2}, y_{3}, \dot{\bar{a}}_{2}, \dot{\bar{b}}_{2}, \dot{\bar{c}}_{2}, \bar{a}_{2}, \bar{b}_{2}, \bar{c}_{2}, \\
& u_{1}, u_{2}, u_{3}, \dot{e}_{1}, \dot{e}_{2}, \dot{e}_{3}, \dot{y}_{1}, \dot{y}_{2}, \dot{y}_{3} \in L_{\infty} .
\end{aligned}
$$

The proof of the projective synchronization of the Lorenz system and the Lü system is thus completed.

\section{Numerical Simulations}

In this section, numerical simulations for the projective synchronization of the systems (20) and (22) are given to demonstrate the effectiveness and feasibility of the proposed controllers (28) and the online parameter update law (35). All the differential equations are solved by using the fourth-order Runge-Kutta method, and step size is set to 0.001 .

In the simulations, parameters $a_{1}, b_{1}, c_{1}$ and the initial conditions of the Lorenz system are chosen as 10, 8/3, 28, 


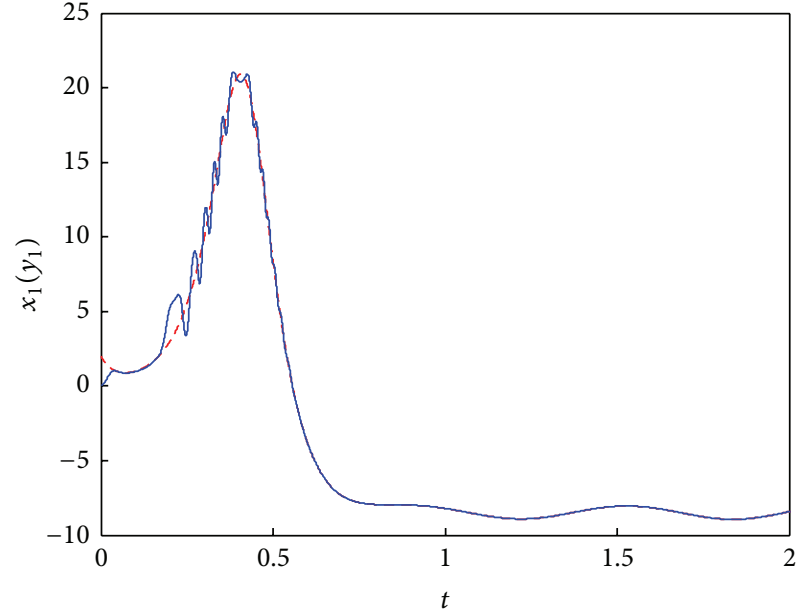

(a)

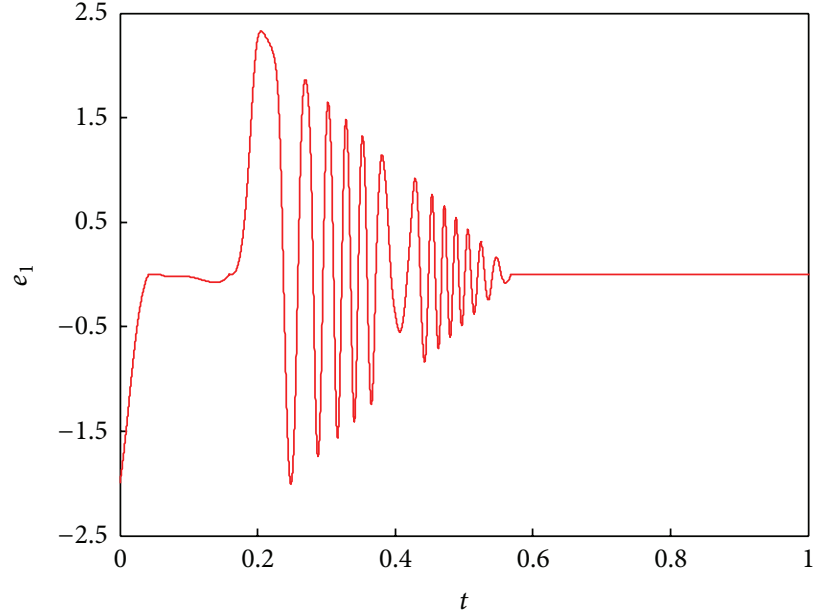

(b)

FIgURE 1: Time series and synchronization error of trajectories of the Lorenz system and the Lü system: (a) $x_{1}\left(y_{1}\right)$ versus $t$; (b) $e_{1}$ versus $t$.

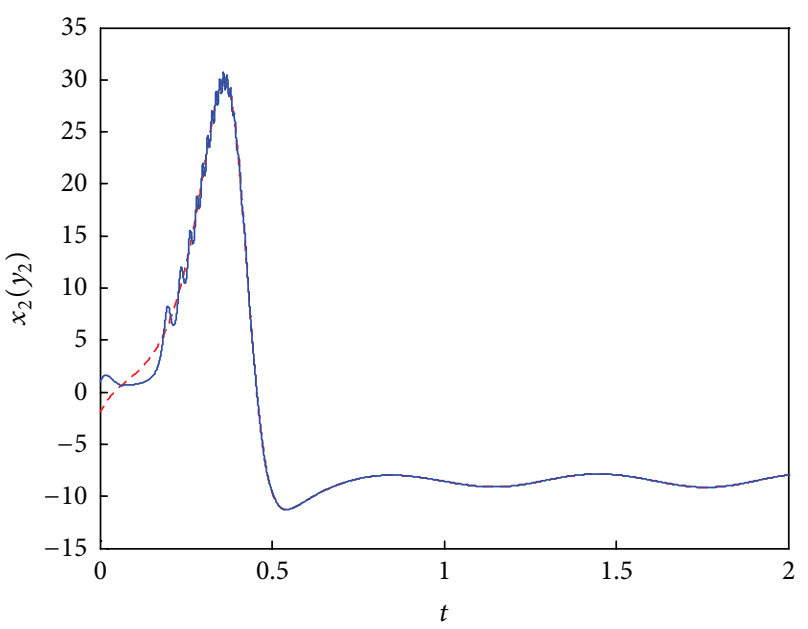

(a)

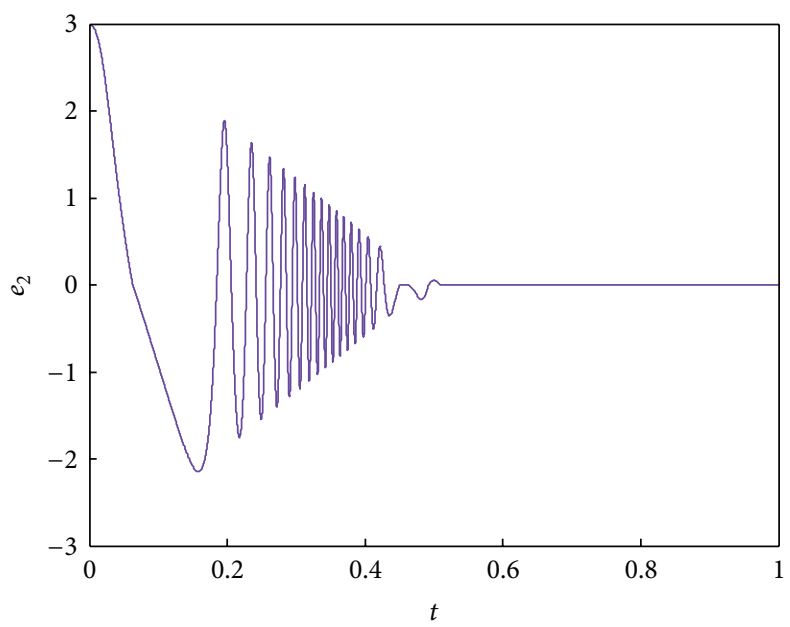

(b)

FIGURE 2: Time series and synchronization error of trajectories of the Lorenz system and the Lü system: (a) $x_{2}\left(y_{2}\right)$ versus $t$; (b) $e_{2}$ versus $t$.

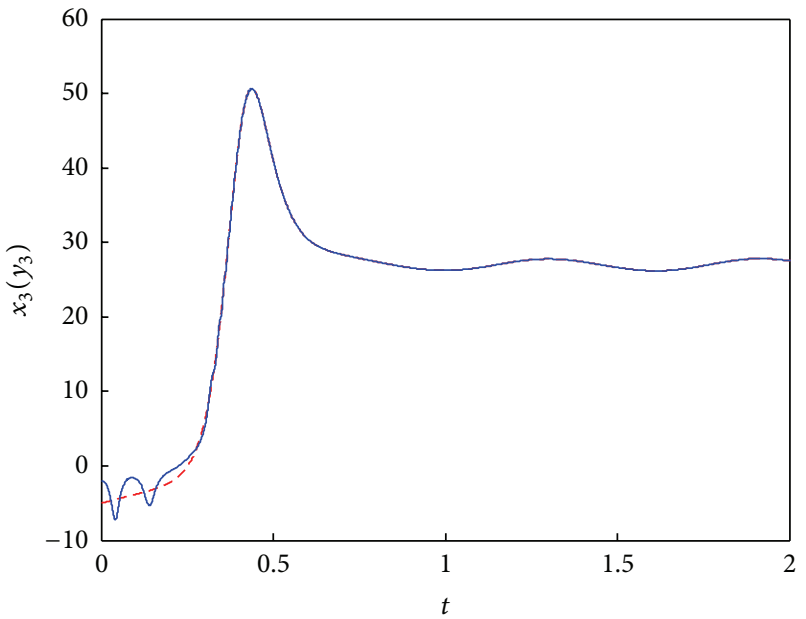

(a)

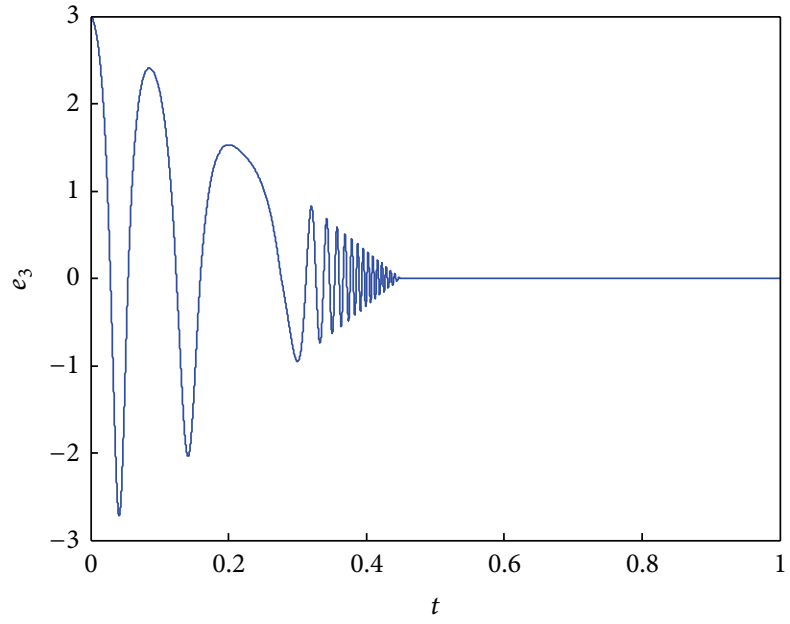

(b)

FIgURE 3: Time series and synchronization error of trajectories of the Lorenz system and the Lü system: (a) $x_{3}\left(y_{3}\right)$ versus $t$; (b) $e_{3}$ versus $t$. 


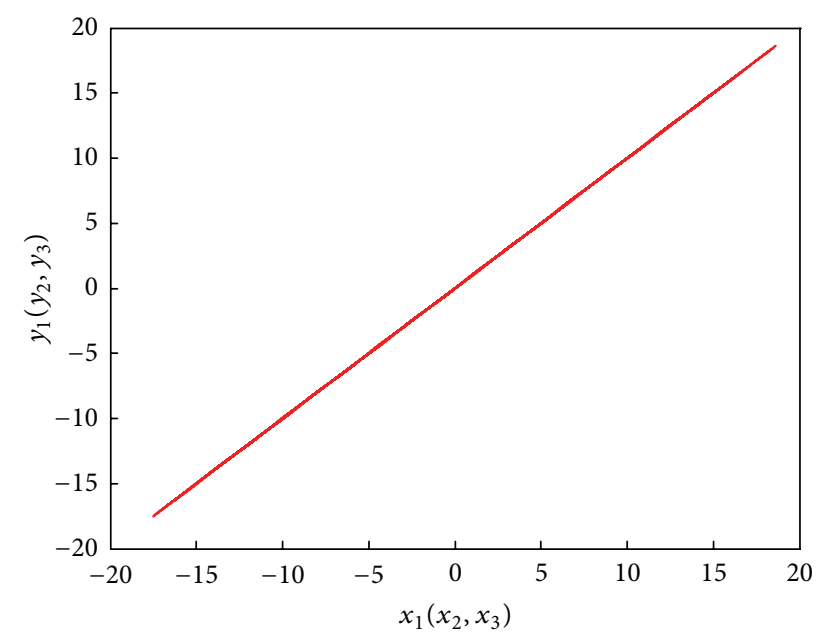

FIGURE 4: Synchronization behavior of the Lorenz system and the Lü system.

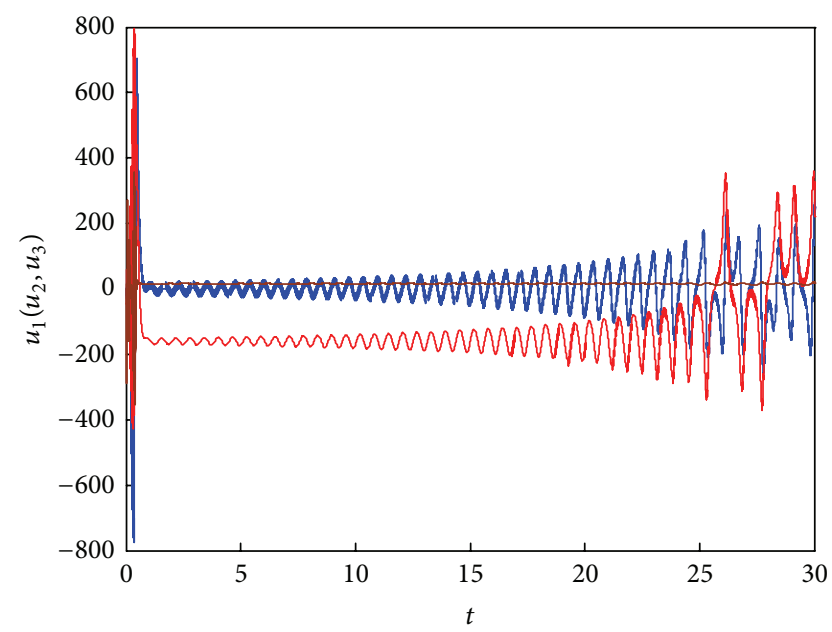

FIGURE 5: Time series of control input (28).

and $x_{0}=(2,-2,-5)$, respectively. Parameters $a_{2}, b_{2}, c_{2}$ and the initial conditions of the Lü system are set to $35,7,2.92$, and $y_{0}=(0,1,-2)$, respectively, and other parameters are

$$
\begin{gathered}
{\left[\begin{array}{l}
\Delta g_{1}(y, \theta, t) \\
\Delta g_{2}(y, \theta, t) \\
\Delta g_{3}(y, \theta, t)
\end{array}\right]=\left[\begin{array}{c}
0.2 \sin (t) \\
0.5 \sin (3 t) \\
0.3 \sin (2 t)
\end{array}\right],} \\
{\left[\begin{array}{l}
d_{1}(t) \\
d_{2}(t) \\
d_{3}(t)
\end{array}\right]=\left[\begin{array}{l}
0.5 \sin (5 t) \\
0.3 \sin (2 t) \\
0.1 \sin (4 t)
\end{array}\right],} \\
\Gamma=\left[\begin{array}{ccc}
20 & 0 & 0 \\
0 & 20 & 0 \\
0 & 0 & 20
\end{array}\right], \\
\eta=9, \quad \kappa=1, \quad \rho(t)=10 .
\end{gathered}
$$

Figures 1(a), 2(a), and 3(a) display the synchronized state trajectories between the Lorenz system and the Lü system.

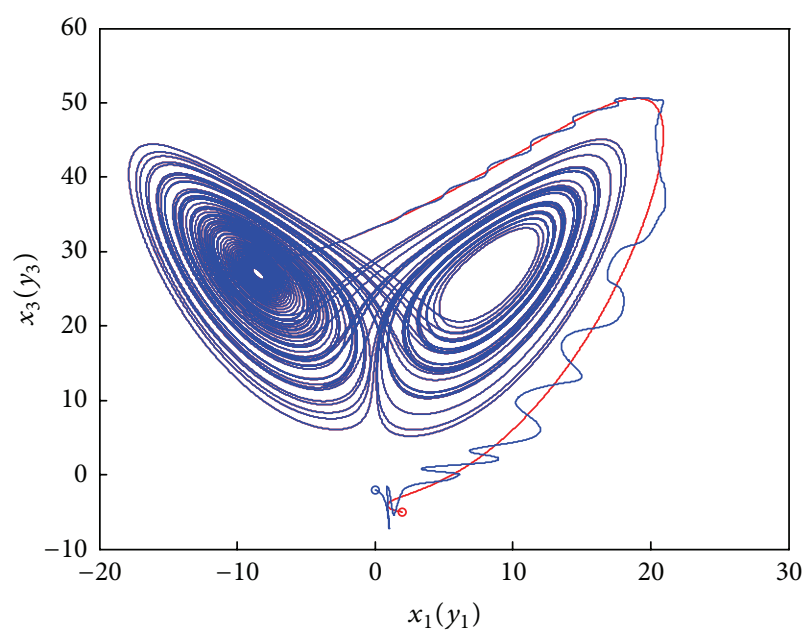

FIGURE 6: Attractors of complete projective synchronization with different conditions between the Lorenz system and the Lü system.

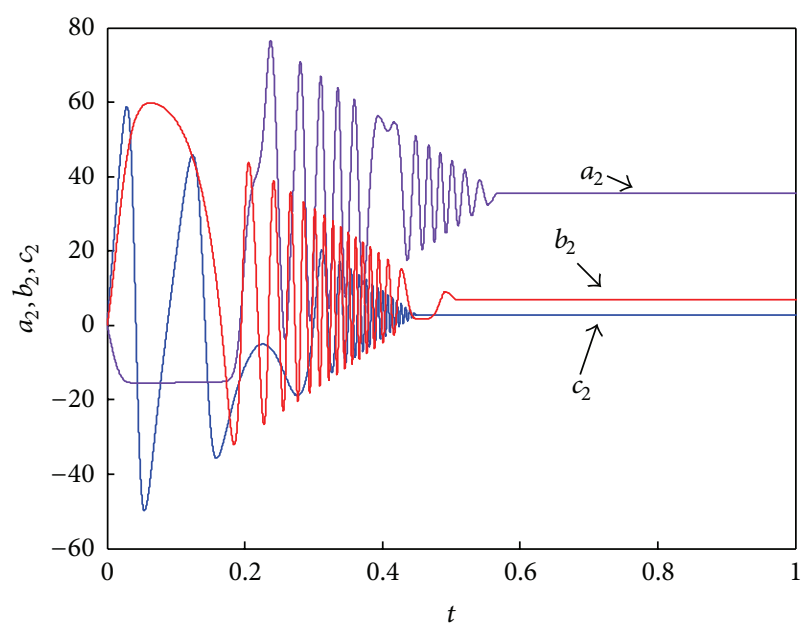

FIGURE 7: The estimated value of the unknown parameters $a_{2}, b_{2}$, and $c_{2}$ in the Lü system.

From the evolution of the state variables, it can be found that the different trajectories from the initial values $x_{0}$ and $y_{0}$ are gradually close to each other and will overlap ultimately. Figures 1(b), 2(b), and 3(b) display that the errors converge to zero as time increases, which implies that the projective synchronization has been realized. Figure 4 shows that the relation curve is a line with a slop of $45^{\circ}$, which illustrates that the trajectories generated from the Lü system will equal that from the Lorenz system.

Figure 5 is the time series of control inputs $u_{1}, u_{2}$, and $u_{3}$, whose main role is to ensure that the response of the Lü system is the same as that of the Lorenz system with the lapse of time. Figure 6 is the projection of the completely synchronized attractors of the Lorenz system and the Lü system on phase plane. The trajectories of the driving system and the response system from different conditions will converge to an identical attractor. Figure 7 depicts the time 


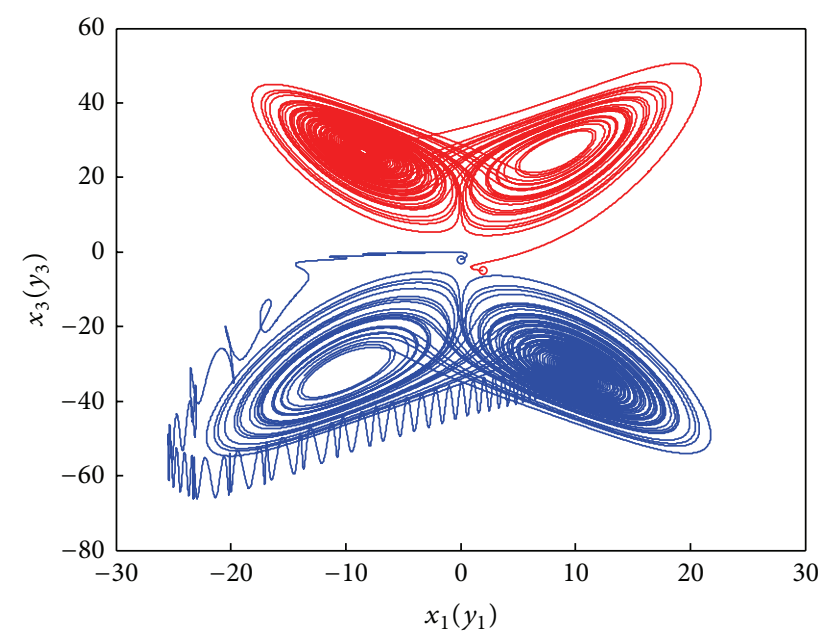

FIGURE 8: Antiphase projective synchronization in proportion with the scaling factor $\kappa=-1.2$.

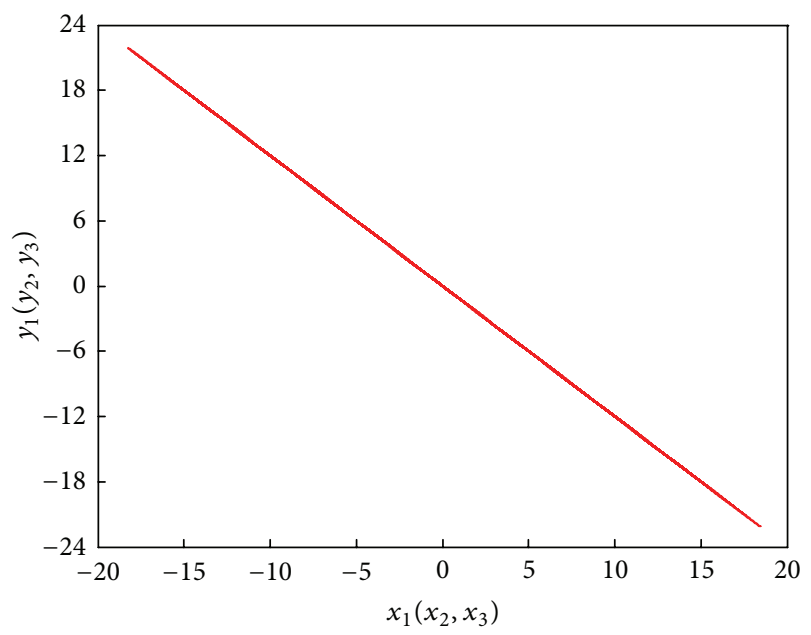

Figure 9: Synchronization behavior of the Lorenz system and the Lü system with the scaling factor $\kappa=-1.2$.

series of the estimated parameter, which are frozen at $a_{2}=35$, $b_{2}=7$, and $c_{2}=2.92$, respectively.

When the scaling factor $\kappa=-1.2$, using the control of inputs $u_{1}, u_{2}$, and $u_{3}$ and the parameter update law (35), the antiphase projection of synchronized attractors on $x_{1}-x_{3}\left(y_{1}-y_{3}\right)$ plane and synchronization behavior of the Lorenz system and the Lü system are shown in Figures 8 and 9, respectively. It is obvious that the antiphase projective synchronization in proportion is obtained by varying the scaling factor $\kappa$.

\section{Conclusions}

In this paper, a projective synchronization theorem was proposed based on an adaptive sliding mode control algorithm when the parameters of the response system are unknown and there are uncertain terms and external bounded disturbances in the response system. As an application of this projective synchronization theorem, the projective synchronization of the Lorenz system and the Lü system with uncertain terms and external bounded disturbances was also analyzed. The globally projective synchronization was achieved by a sliding mode controller based on the adaptive technique. The numerical simulations demonstrated the effectiveness and feasibility of the proposed technique. For the proposed method, there are potential applications in higher speed secure digital communication. In the future, we will try to extend the proposed method to the consensus of multiagent systems such as the networked chaotic systems and complex networks.

\section{Acknowledgments}

This work was supported in part by the National Natural Science Foundation of China (nos. 61174094 and 11202148), China/South Africa Research Cooperation Programme (nos. 78673 and CS06-L02), and South African National Research Foundation Incentive Grant (no. 81705).

\section{References}

[1] E. Mosekilde, Y. Maistrenko, and D. Postnov, Chaotic Synchronization: Applications to Living Systems, vol. 42, World Scientific Publishing Company, River Edge, NJ, USA, 2002.

[2] L. M. Pecora, T. L. Carroll, G. A. Johnson, D. J. Mar, and J. F. Heagy, "Fundamentals of synchronization in chaotic systems, concepts, and applications," Chaos, vol. 7, no. 4, pp. 520-543, 1997.

[3] G. Alvarez, L. Hernández, J. Muñoz, F. Montoya, and S. Li, "Security analysis of communication system based on the synchronization of different order chaotic systems," Physics Letters A, vol. 345, no. 4-6, pp. 245-250, 2005.

[4] A. Khadra, X. Liu, and X. Shen, "Application of impulsive synchronization to communication security," IEEE Transactions on Circuits and Systems. I. Fundamental Theory and Applications, vol. 50, no. 3, pp. 341-351, 2003.

[5] Q. Xie, G. Chen, and E. M. Bollt, "Hybrid chaos synchronization and its application in information processing," Mathematical and Computer Modelling, vol. 35, no. 1-2, pp. 145-163, 2002.

[6] L. A. Mozelli, C. D. Campos, R. M. Palhares, L. A. B. Tôrres, and E. M. A. M. Mendes, "Chaotic synchronization and information transmission experiments: a fuzzy relaxed Ho control approach," Circuits, Systems, and Signal Processing, vol. 26, no. 4, pp. 427-449, 2007.

[7] H. G. Enjieu Kadji, J. B. Chabi Orou, and P. Woafo, "Synchronization dynamics in a ring of four mutually coupled biological systems," Communications in Nonlinear Science and Numerical Simulation, vol. 13, no. 7, pp. 1361-1372, 2008.

[8] S. Xu and Y. Yang, "Global asymptotical stability and generalized synchronization of phase synchronous dynamical networks," Nonlinear Dynamics, vol. 59, no. 3, pp. 485-496, 2010.

[9] D. Y. Tang, R. Dykstra, M. W. Hamilton, and N. R. Heckenberg, "Observation of generalized synchronization of chaos in a driven chaotic system," Physical Review E, vol. 57, no. 5, pp. 5247-5251, 1998. 
[10] G. Álvarez, F. Montoya, G. Pastor, and M. Romera, "Breaking a secure communication scheme based on the phase synchronization of chaotic systems," Chaos, vol. 14, no. 2, pp. 274-278, 2004.

[11] G. M. Mahmoud and E. E. Mahmoud, "Phase and antiphase synchronization of two identical hyperchaotic complex nonlinear systems," Nonlinear Dynamics, vol. 61, no. 1-2, pp. 141-152, 2010.

[12] M.-C. Ho, Y.-C. Hung, and C.-H. Chou, "Phase and anti-phase synchronization of two chaotic systems by using active control," Physics Letters A, vol. 296, no. 1, pp. 43-48, 2002.

[13] M. Mossa Al-sawalha and M. S. M. Noorani, "On anti-synchronization of chaotic systems via nonlinear control," Chaos, Solitons and Fractals, vol. 42, no. 1, pp. 170-179, 2009.

[14] G. M. Mahmoud and E. E. Mahmoud, "Complete synchronization of chaotic complex nonlinear systems with uncertain parameters," Nonlinear Dynamics, vol. 62, no. 4, pp. 875-882, 2010.

[15] X.-F. Li, A. C.-S. Leung, X.-P. Han, X.-J. Liu, and Y.-D. Chu, "Complete (anti-)synchronization of chaotic systems with fully uncertain parameters by adaptive control," Nonlinear Dynamics, vol. 63, no. 1-2, pp. 263-275, 2011.

[16] A. Englert, W. Kinzel, Y. Aviad et al., "Zero lag synchronization of chaotic systems with time delayed couplings," Physical Review Letters, vol. 104, no. 11, Article ID 114102, 2010.

[17] D. Pazó, M. A. Zaks, and J. Kurths, "Role of unstable periodic orbits in phase and lag synchronization between coupled chaotic oscillators," Chaos, vol. 13, no. 1, pp. 309-318, 2003.

[18] R. Mainieri and J. Rehacek, "Projective synchronization in three-dimensional chaotic systems," Physical Review Letters, vol. 82, no. 15, pp. 3042-3045, 1999.

[19] X.-Y. Wang and M.-J. Wang, "Projective synchronization of nonlinear-coupled spatiotemporal chaotic systems," Nonlinear Dynamics, vol. 62, no. 3, pp. 567-571, 2010.

[20] F. Farivar, M. A. Shoorehdeli, M. A. Nekoui, and M. Teshnehlab, "Chaos control and modified projective synchronization of unknown heavy symmetric chaotic gyroscope systems via Gaussian radial basis adaptive backstepping control," Nonlinear Dynamics, vol. 67, no. 3, pp. 1913-1941, 2012.

[21] D. Xu, Z. Li, and S. R. Bishop, "Manipulating the scaling factor of projective synchronization in three-dimensional chaotic systems," Chaos, vol. 11, no. 3, pp. 439-442, 2001.

[22] G. Wen and D. Xu, "Nonlinear observer control for fullstate projective synchronization in chaotic continuous-time systems," Chaos, Solitons and Fractals, vol. 26, no. 1, pp. 71-77, 2005.

[23] K. S. Sudheer and M. Sabir, "Function projective synchronization in chaotic and hyperchaotic systems through open-plusclosed-loop coupling," Chaos, vol. 20, no. 1, Article ID 013115, 2010.

[24] G. Peng and Y. Jiang, "Generalized projective synchronization of a class of fractional-order chaotic systems via a scalar transmitted signal," Physics Letters A, vol. 372, no. 22, pp. 3963-3970, 2008.

[25] C. Y. Chee and D. Xu, "Secure digital communication using controlled projective synchronisation of chaos," Chaos, Solitons and Fractals, vol. 23, no. 3, pp. 1063-1070, 2005.

[26] C. Sarasola, F. J. Torrealdea, A. D’Anjou, A. Moujahid, and M. Graña, "Feedback synchronization of chaotic systems," International Journal of Bifurcation and Chaos in Applied Sciences and Engineering, vol. 13, no. 1, pp. 177-191, 2003.
[27] R. Femat, J. Alvarez-Ramírez, and G. Fernández-Anaya, "Adaptive synchronization of high-order chaotic systems: a feedback with lower-order parametrization," Physica D, vol. 139, no. 3-4, pp. 231-246, 2000.

[28] A. Khadra, X. Liu, and X. Shen, "Impulsive control and synchronization of spatiotemporal chaos," Chaos, Solitons and Fractals, vol. 26, no. 2, pp. 615-636, 2005.

[29] Y. Chai and L.-Q. Chen, "Projective lag synchronization of spatiotemporal chaos via active sliding mode control," Communications in Nonlinear Science and Numerical Simulation, vol. 17, no. 8, pp. 3390-3398, 2012.

[30] S. Cang, G. Qi, and Z. Chen, "A four-wing hyper-chaotic attractor and transient chaos generated from a new 4-D quadratic autonomous system," Nonlinear Dynamics, vol. 59, no. 3, pp. 515-527, 2010.

[31] C. Shi-Jian, C. Zeng-Qiang, and W. Wen-Juan, "Circuit implementation and multiform intermittency in a hyper-chaotic model extended from the Lorenz system," Chinese Physics B, vol. 18, no. 5, pp. 1792-1800, 2009.

[32] D. Lin and X. Wang, "Observer-based decentralized fuzzy neural sliding mode control for interconnected unknown chaotic systems via network structure adaptation," Fuzzy Sets and Systems, vol. 161, no. 15, pp. 2066-2080, 2010.

[33] C. Mou, C.-S. Jiang, J. Bin, and Q.-X. Wu, "Sliding mode synchronization controller design with neural network for uncertain chaotic systems," Chaos, Solitons and Fractals, vol. 39, no. 4, pp. 1856-1863, 2009.

[34] Y.-S. Yang, J.-F. Chang, T.-L. Liao, and J.-J. Yan, "Robust synchronization of fractional chaotic systems via adaptive sliding mode control," International Journal of Nonlinear Sciences and Numerical Simulation, vol. 10, no. 9, pp. 1237-1244, 2009.

[35] E. N. Lorenz, "An attractor embedded in the atmosphere," Tellus $A$, vol. 58, no. 4, pp. 425-429, 2006.

[36] J. Lü and G. Chen, "A new chaotic attractor coined," International Journal of Bifurcation and Chaos in Applied Sciences and Engineering, vol. 12, no. 3, pp. 659-661, 2002. 


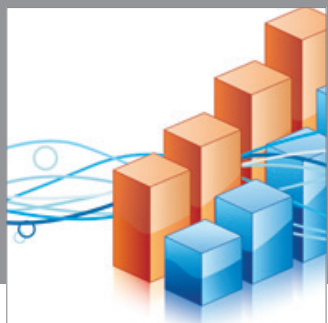

Advances in

Operations Research

mansans

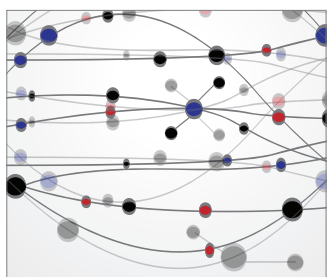

The Scientific World Journal
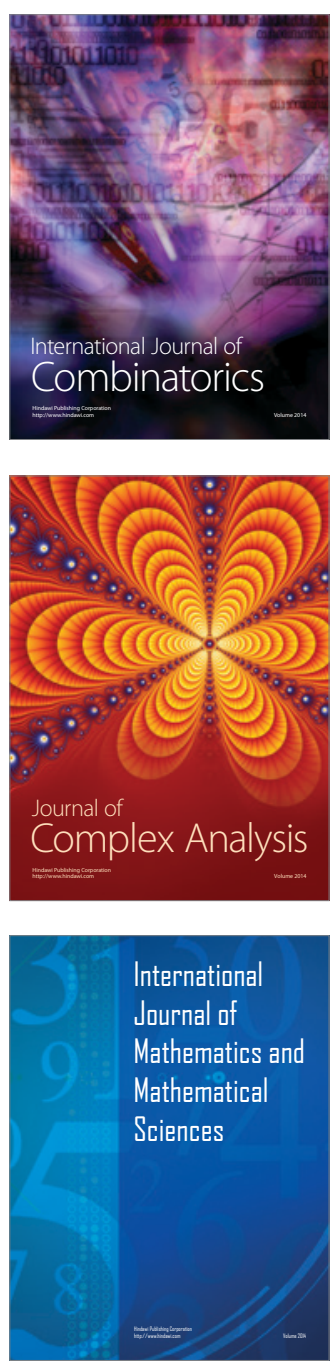
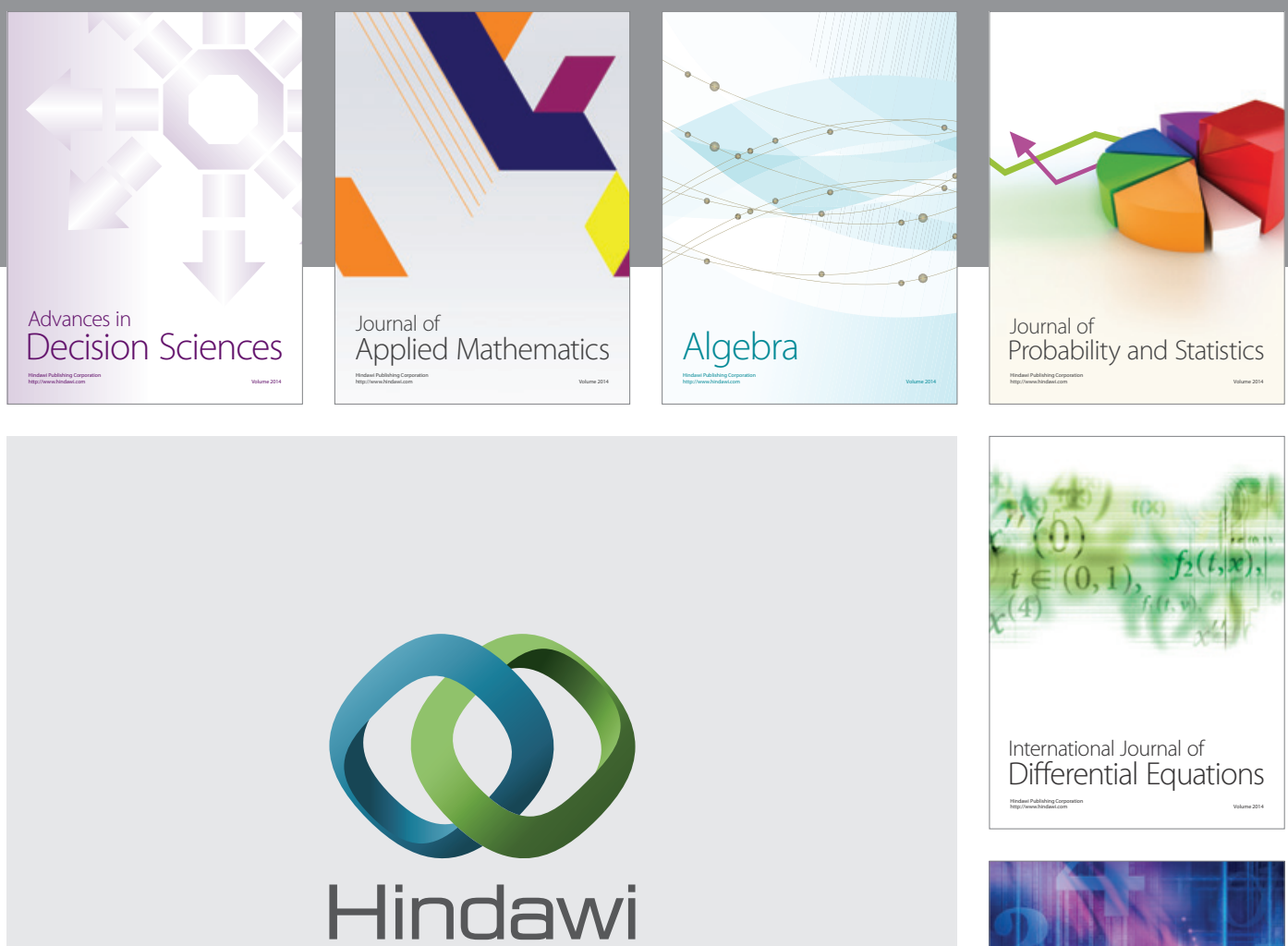

Submit your manuscripts at http://www.hindawi.com
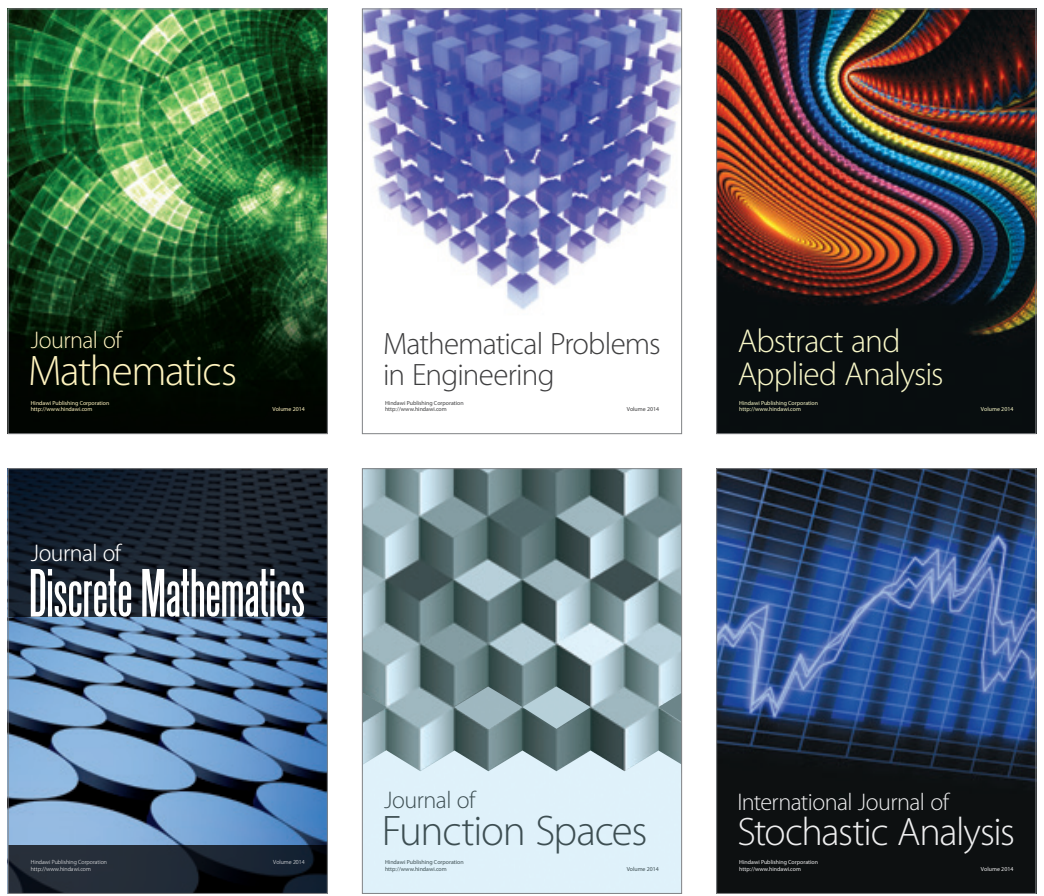

Journal of

Function Spaces

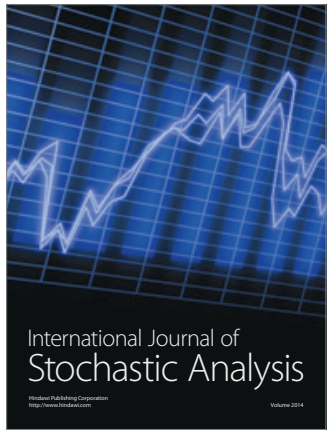

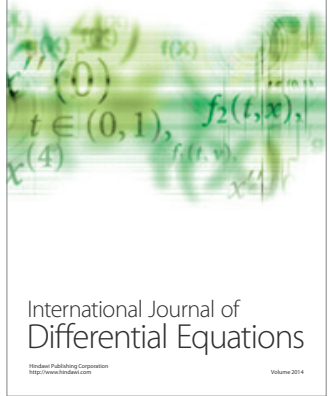
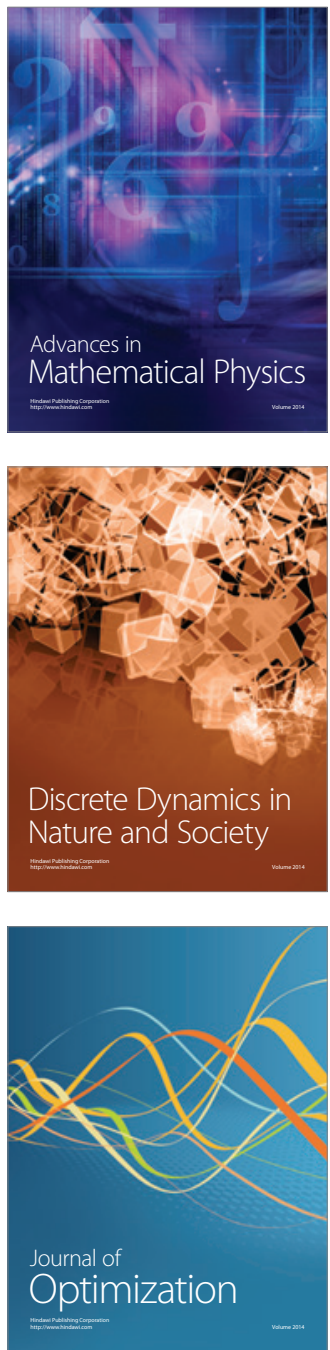